\title{
The Course Design of Android Location Service
}

\author{
Jihong CHENG, Zhen WANG, Xiuli TAN \\ Yantai Nanshan University, Yantai Shandong, China
}

\begin{abstract}
Location service is a special function in the Android OS, LBS app is an import field in the mobile application development. Using Baidu map SDK as a tool, the Android location service teaching course which comply with the Model-Driven teaching method in pursuit of teaching process repeatability, operability and easy to be accepted. Finally, the answers of the problems encountered in the teaching are given. The problem that the Google map sdk is difficult to download and the key is difficult to apply is solved. KEYWORD: LBS; Android; Location service; Teaching design
\end{abstract}

With the development of smart phone applications such as home appliances, mobile application development is experiencing a period of rapid development. Android as a free mobile operating system is favored by most mobile developer and manufacturer. In order to meet business needs, all colleges and universities have set up Android mobile development courses. How to develop Android mobile teaching becomes a hot issue in modern teaching. As a smart phone features, Maps and location-based services become the highlight of APP. As the network reasons Google Maps SDK cannot be downloaded, Key difficult to apply, it is not in line with Chinese applications, so choose Baidu map as a teaching tool to develop the locationbased services of Android. The content arrangement and development steps are loyally obey to textbook. Finally the App demo is finished and runs successfully. Trial teaching by Computer Science and Technology students (2012) is proved to be success. The course design is good to understanding by students.

The instructional design using case-driven teaching methods, refinement steps, each step are given detailed operational description and code. For the case of the choice to comply with the Cheng \& Lou (2014) literature requirements, as simple as possible to avoid complex class relations, thus avoiding the students trap into understanding the case itself rather than teaching contents.

\section{INTRODUCTION OF BAIDU MAP}

Before Baidu map development, briefly explain
Baidu map. Baidu map provide a wealth of features, the map display, POI search, geocoding and reverse, road planning, map overlay, location, offline maps, navigation and LBS cloud and other features (The map display, geocoding and reverse, map overlay, location and navigation are required teaching contents), described as follows.

\subsection{Map display and operation}

Baidu map can be displayed in $2 \mathrm{D}$ or $3 \mathrm{D}$, satellite maps and real-time traffic mode. Operation via interface (programming), gestures to achieve the map click, double click, long press, bloom, rotate and change the perspective of map, etc.

\subsection{POI search}

Periphery searching, domain searching and city searching are supported. Periphery searching is that according to the distance and keywords specified by the user and a center make the POI searching. Domain searching is that in a rectangular area make the POI searching with keywords. City searching is that searches with keywords in a city. POI detail searching also supported, namely based on the POI ID retrieves the detail of the interest point.

\subsection{Geocoding and reverse geocoding}

Geocoding refers to the mutual conversion between geographic coordinates and address. Geocoding achieve the Chinese address or place name converting to the coordinates on the Earth's surface, the reverse geocoding achieve the geo-coordinates 
converting to address or place name.

\subsection{Road planning}

Public transportation query, bus transfer, driving route planning and walking route searching are supported by Baidu map.

\subsection{Map overlay}

Baidu Map SDK supports a variety of map overlay to help you show richer maps. Currently supported map covering are: positioning layers, map annotation (Marker), geometry (points, polylines, arcs, polygons, etc.), topographic map layers, POI search results layers, route planning results layers, thermodynamic chart layers and so on.

\subsection{Location}

Using GPS, WIFI, base stations, IP hybrid positioning mode, Baidu map use the Android SDK positioning obtain location information and use map SDK location layers displaying the result.

\subsection{Offline map}

Users can import offline map pack by two forms of manual and interface SDK, offline map can save user traffic, providing better map display effect.

\subsection{Navigation}

SDK currently supports client navigation and Web page navigation (H5 navigation). It is noteworthy that using Baidu map navigation, you need to install Baidu map version 5.0 and above on the device.

\subsection{LBS cloud}

LBS cloud is a new platform-service for LBS developers, not only for PC applications development, but also for mobile devices. Mobile developers using LBS cloud server can be achieved at no cost to store vast amounts of data and maintenance, and supports efficient retrieval of user data to achieve the map show.

In addition to these functions, Baidu map also provides the following features: a short string share, Place and exhibition details page. Short string Share: The POI search results or reverse geocoding results generate short string, when other users click on a short string the map view is displayed via Baidu client or browser of mobile. Place retrieve details: According to the POI ID information, retrieve the details of the POI. Thermodynamic chart feature: open thermodynamic mapping capabilities to help developers to build their own thermodynamic chart.

\section{DEVELOPING ENVIRONMENT}

OS: Windows XP SP3;

IDE: adt-bundle-windows-x 86-20140321;

Android SDK: 4.4.2 (API19);

Baidu map: 3.2.0.

Emulator: Emulator bundled with IDE.

\section{TEACHING DESIGN}

\subsection{Obtaining debug certificate}

In Eclipse window menu select Preferences option, in the dialog window select the Android node and Build option. In the textbox after SHA1 fingerprint is the signature fingerprint. In this case, you can use the default signature fingerprint.

\subsection{Obtaining the Baidu developer key}

Enter the URL http://lbsyun.baidu.com/apiconsole/key in the browser address. Click the Create Application button in open the page. Enter the application name and select the application type (here the choice for server must be selected, otherwise the map cannot be displayed), in the IP white list enter 0.0.0.0/0 that indicate the server calls unlimited, click submit button to complete the key application. At this point, you need to pay attention to that, with Baidu map upgrade, the new version and the old version key application has some differences. The method described above is the new version application method.

\subsection{Create Android project}

Create Android project and name it LBS. First, download the Baidu map SDK from http://developer.baidu.com/map/index.php?title=and roidsdk/sdkandev-download, then unzip the BaiduLBS_AndroidSDK_Lib.zip file and copy all files (armeabi directory, android_api_1.1_forsdk.jar, BaiduLBS_Android.jar and galaxy_mini.jar) under lib directory to libs directory of Android project. Now the project structure is like figure 1. 


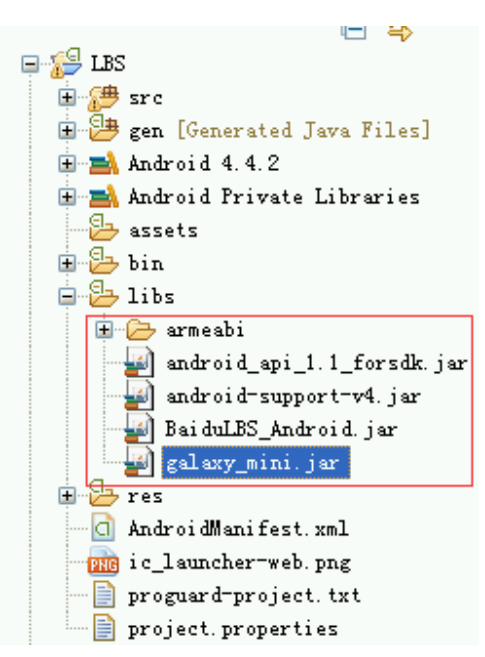

Figure 1 Baidu map project structure

Right click project name LBS, select Build Path $\rightarrow$ Configure Build path... from popup menu, at Order and Export tab in the dialog window check the Android Private Libraries and Android Dependencies options, then at the Libraries tab click the Add External JARs button, browse the android_api_1.1_forsdk.jar, BaiduLBS_Android.jar, galaxy_mini.jar files and reference them. Finally, clean the project and build again.

\subsection{Add map key}

In the <application/> node of LBS AndroidManifest.xml file add the following code: <meta-data android: name="com. baidu. lbsapi. API_KEY"

android:

value="ez4osEVazh8QXqqdAQz9PRko" />

The android: value is the key applied in 3.2 section. The android: name's value is com. baidu. lbsapi. API_KEY which is predefined and cannot be changed. The android: value property needs change to his key; otherwise the map cannot be displayed. In this case, the student should be noted that: android: value attribute value to apply to his own developer key, but cannot copy the key of the teaching projects.

\subsection{Add Android permission}

In the AndroidManifest.xml file enter the Baidu map permission, as the following:

<uses-permission android: name="android. permission. GET_ACCOUNTS" />

<uses-permission android: name $=$ "android. permission. USE_CREDENTIALS" />

<uses-permission android: name="android. permission. MANAGE_ACCOUNTS" / >

<uses-permission android: name $=$ "android. permission. AUTHENTICATE_ACCOUNTS" />

<uses-permission android: name="android. permission. ACCESS_NETWORK_STATE" />

<uses-permission android: name="android. permission. INTERNET" /> <uses-permission android: name="com. android.

launcher. permission. READ_SETTINGS" / >

<uses-permission android: name="android.

permission. CHANGE_WIFI_STATE" / >

<uses-permission android: name="android.

permission. ACCESS_WIFI_STATE" />

<uses-permission android: name="android.

permission. READ_PHONE_STATE" />

<uses-permission android: name="android.

permission. WRITE_EXTERNAL_STORAGE" />

<uses-permission android: name="android.

permission. BROADCAST_STICKY" />

<uses-permission android: name="android.

permission. WRITE_SETTINGS" />

<uses-permission android: name="android. permission. READ_PHONE_STATE" />

\subsection{Add the Baidu map view}

Add the following code into the activity_lbs.xml layout file. Map View is the view to display the map. The id attribute is the ID for referencing the map view. The layout_width and layout_height attribute control the map view's width and length. The clickable attribute indicates that the map response to the user click event or not, which is true indicates the map response the user click event.

<com. baidu. mapapi. map. Map View android: id="@+id/id_bmap View" android: layout_width="fill_parent" android: layout_height="fill_parent" android: clickable="true" />

\subsection{The map control}

\subsubsection{Initializing the map}

In the on Create() method, add the following statements:

@Override

protected void on Create (Bundle saved Instance

State) \{

super. On Create (saved Instance State); request Window Feature (Window.

FEATURE_NO_TITLE); SDK Initializer. initialize (get Application Context());

set Content View(R. layout. activity_lbs); $\mathrm{m}$ Map View $=($ Map View $)$ find View ById $(\mathrm{R}$. id. id_bmap View);

\}

The request Window Feature() method is used to set the Activity window display mode, the Windows. FEATURE_NO_TITLE constant indicates that the window has no title, so saving space for map display. The SDK Initializer. Initialize (Application Context) method accept the Application Context object and initialize the map. 


\subsubsection{Map lifecycle control}

Since the map is very wasted of power and resources, it is necessary to control the map life cycle. When the Activity turn to the background and the Activity object is destroyed we should terminate the map display and release the map object. So we add following statements to the on Destroy (), on Resume () and on Pause () method as follows:

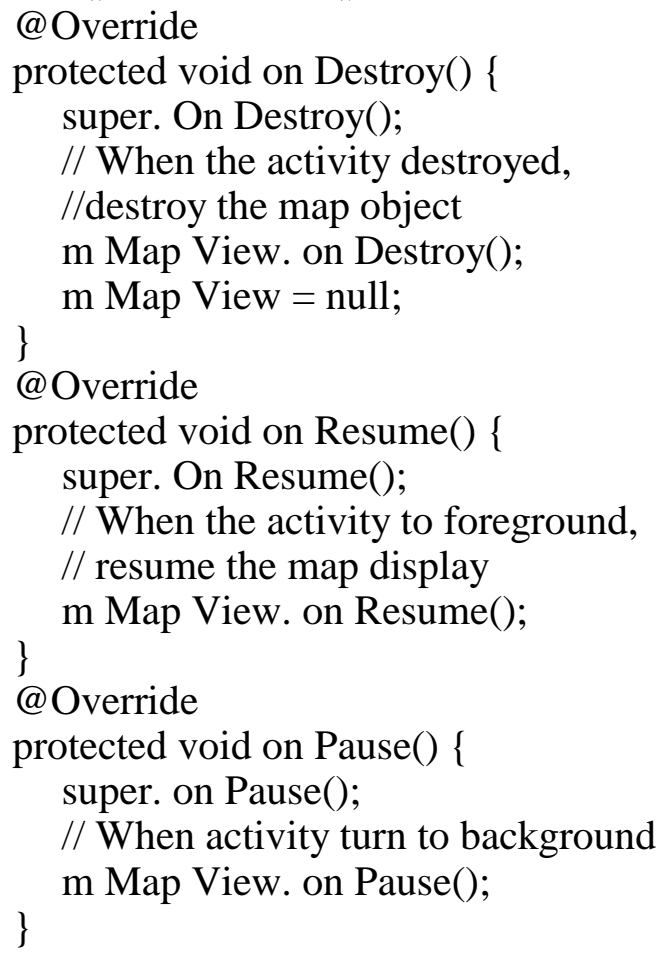

\subsection{Testing the App}

Select Android Virtual Device Manager in the Eclipse Window menu, in the popup dialog window select the custom simulator and click Start button to open the simulator. In the Package Explorer, rightclick the project name LBS and choose Run as ... $\rightarrow$ Android Application. Wait a minute the map will display. In this case, note that: (1) Emulator start very slowly, in order to avoid wasting precious classroom time, remind students start Emulator before teaching. (2) Some emulator isn't good at displaying the map (eg Blue Stack emulator). Map display shown in Figure 2.

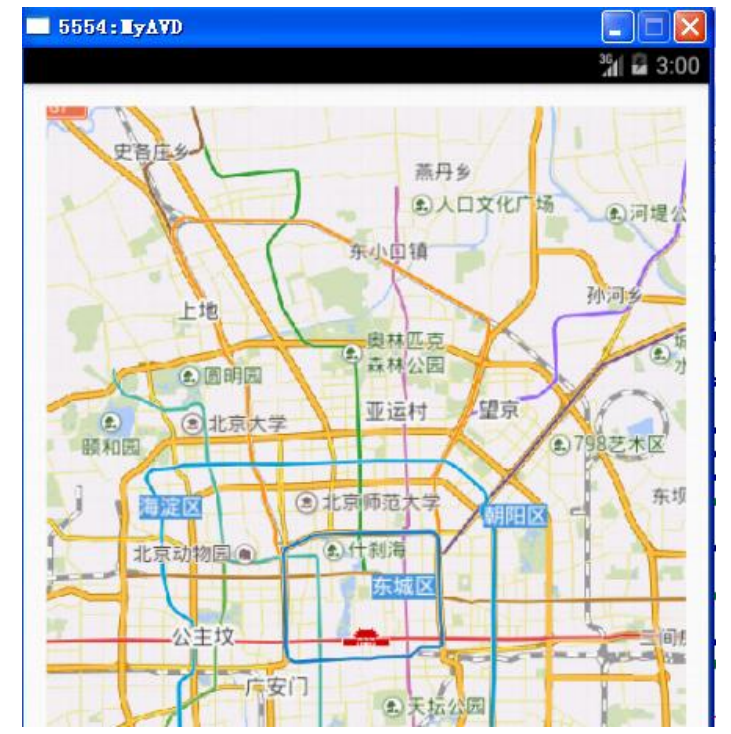

Figure 2 Displaying the map

\section{DISCUSSION}

The most problems encountered in teaching is that the map cannot display. The cause of the problem: (1) The AndroidMenifest.xml file Baidu map key configuration error (see 3.4 Add map key); (2) In the AndroidManifest.xml didn't add INTERNET permission (see 3.5 Add Android permission); (3) Emulator is not connected to the network.

\section{CONCLUSION}

This article discusses the teaching design of Android location services with Baidu Map. This design loyally obeys the textbook arrangement. Solve the difficulties of Google map SDK download and the key application. Mainly from the map display, map operations, obtain location data and other aspects of the teaching content and procedures were designed. By trial of Computer Science and Technology students (2012) which will help students master the learning content. More applications such as zooming map, mode conversion, navigation, add map layer, obtain the map position, geocoding and reverse geocoding, etc. due to limited space, not discussed here.

\section{REFERENCES}

[1] http://blog.csdn.net/lmj623565791/article/details/3772909 1

[2] http://developer.baidu.com/map/index.php?title=androids $\mathrm{dk}$

[3] Cheng, J. H. \& Lou, S. Y. 2014. Application of CaseStudy teaching with recursive case. Computer programming skills \& maintenance (2014(8)):153-155

[4] Lee, W. M. 2012. Beginning Android4 Application Development. Indianapolis: John Wiley \& Sons, Inc. 